\title{
Marking estimation of Petri nets with pairs of nondeterministic transitions
}

\author{
Alessandro Giua, Carla Seatzu \\ Dipartimento Ingegneria Elettrica ed Elettronica \\ Università di Cagliari,Italy \\ e-mail: \{giua,seatzu\}@diee.unica.it \\ Jorge Júlvez \\ Departamento de Informática e Ingeniería de Sistemas \\ Universidad de Zaragoza, Spain \\ e-mail: julvez@unizar.es
}

\begin{abstract}
We present a technique for estimating the marking of a Petri net based on the observation of transition labels. In particular, the main contribution of the paper consists in deriving a methodology that can handle the case of nondeterministic transitions, i.e., transitions that share the same label. Under some technical assumptions, the set of markings consistent with an observation can be represented by a linear system with a fixed structure that does not depend on the length of the observed word.
\end{abstract}

Keywords: Petri nets, observability, marking estimation, nondeterministic transitions.

\section{Introduction}

This paper deals with the problem of estimating the marking of a Place/Transition $(\mathrm{P} / \mathrm{T})$ net based on the observation of transition firings. The problem of estimating the state of a dynamic system is a fundamental issue in system theory and the construction of state observers for timedriven systems is treated in most linear systems textbooks. Although less popular in the case of discrete-event systems, the issue of state estimation under partial state observation has been discussed in the literature. For systems represented as finite automata, Ramadge [12] was the first to show how an observer could be designed for a partially observed system. Caines et al. [2] 
showed how it is possible to use the information contained in the past sequence of observations (given as a sequence of observation states and control inputs) to compute the set of consistent states, while in [3] the observer output is used to steer the state of the plant to a desired terminal state. A similar approach was also used by Kumar et al. [7] when defining observer based dynamic controllers in the framework of supervisory predicate control problems. Özveren and Willsky [10] proposed an approach for building observers that allows one to reconstruct the state of finite automata after a word of bounded length has been observed, showing that an observer may have an exponential number of states.

Let us define the set of states consistent with the observed behavior as the states in which the system may actually be given the observation. There are two main drawbacks in the above mentioned automata based approaches to the design of a discrete event observer. Firstly, the set of consistent states must explicitly be enumerated. Secondly, to compute the set of consistent states at step $k$ it is not usually sufficient to know the new observation and the set of consistent states at step $k-1$, but it is necessary to recompute this set as a function of all previous observations.

Looking for more efficient approaches that do not require the enumeration of this set, we explored the possibility of using Petri nets as discrete event models [5, 6]. We showed that under the following three assumptions: (A1') the net structure is known; (A2') the initial marking is not known or is only known to belong to an initial macromarking, i.e., a given linear convex set; (A3') all transition firings can be observed; it is possible to represent the set of consistent markings (i.e., the states of the Petri net) as the solution of a linear system that has a fixed structure which only depends on two parameters (the estimate and the bound) that can be recursively computed. Note that other authors [8] have also discussed the problem of estimating the marking of a Petri net using a mix of transition firing and place observations.

In this paper we further extend the approach of $[5,6]$ relaxing what we felt was its major limitation, i.e., the assumption (A3') that all transition firings can be observed. Given an alphabet of symbols $E$, we assume that a labeling function $L: T \rightarrow E$ assigns to each transition $t$ a label $L(t) \in E$. The labeling function we consider may assign to two or more transitions the same label, i.e., using a common Petri net terminology, it is called a $\lambda$-free labeling.

When $t$ fires, only its label $L(t)$ is observed and this may introduce nondeterminism in the observer, in the sense that the observed word is not sufficient to reconstruct the transition firing and thus the actual marking. Note, however, that in this paper we restrict assumption (A2') assuming that the initial marking is perfectly known. In effect, this may not be strictly necessary but we need it in this paper to simplify the results we present. 
The above mentioned framework naturally applies to all those cases in which the structure of the considered plant is perfectly known and we can also evaluate (measure) its initial configuration. As the system evolves, it is not always convenient (or even possible) to measure the actual configuration of the plant. In fact, it is often the case that the actual configuration can only be reconstructed on the basis of the observation of some events, i.e., on the basis of the output of some sensors. To reduce the number of sensors within the plant, it also commonly occurs that the same sensor is used to measure different types of events, thus obviously loosing some information. As an example, in a manufacturing environment, if the same sensor is used to evaluate the status of an unreliable machine that may process different parts, on the basis of the observation of the output of the sensor we can only evaluate if the machine is operational or not, but in the case that it is operational, we cannot evaluate which kind of part it is working.

In a first part of the paper, we show a rather simple result: using the net state equation it is possible to represent the set of consistent markings as the solution of a linear system that can be recursively computed, but whose structure, unfortunately, is not fixed: it grows linearly with the length of the observed word. A similar approach that uses a logical formalism rather than linear programming was also presented by Benasser [1]. This author has studied the possibility of defining the set of markings reached firing a "partially specified" step of transitions using logical formulas, without having to enumerate this set.

In a second part of the paper, we propose a different approach that, under some technical assumptions, allows us to characterize the set of consistent markings as the solution of a different linear system with a fixed structure that depends on some parameters (the upper bounds $u$ 's) that can recursively be computed. In particular, we consider some restrictions on the structure of the labeling function.

- Firstly, we assume that the same label cannot be assigned to more than two transitions.

- Secondly, we assume that nondeterministic transitions (i.e., transitions whose label is also associated to other transitions) should also be contact free, i.e., if $t$ and $t^{\prime}$ are nondeterministic transitions the set of input and output places of $t$ cannot intersect the set of input and output places of $t^{\prime}$.

In all fairness, we admit that these two restrictions may limit in some cases the generality of the approach. However, we also believe that this paper shows a new original framework for observers design based on linear algebra. The results obtained so far look promising and may pave the way for future extensions.

We summarize the main advantages of the proposed approach with respect to other solutions previously presented in the literature as follows. 
1. The proposed linear algebraic characterization of the set of consistent markings does not require the exhaustive enumeration of the consistent states, as in the case of the automata based approaches.

2. The proposed linear algebraic characterization consists of a finite number of constraints, not depending on the length of the observed word. This is not the case in other approaches such as that one of Benasser [1].

3. We have extended our previous results in [6] relaxing the assumption that all events may be observed.

The paper is structured as follows. In Section 2 some background on Petri nets is provided. In Section 3 the considered problem is formally stated and an introductory example is presented. The first characterization of the set of consistent markings, involving a number of constraints that increases as the length of the observed word increases, is given in Section 4. The main significant contribution of the paper is presented in Section 5 where a numerical example is also presented. In Section 6 conclusions are finally drawn and the goal of the future research is also discussed.

\section{$2 \quad$ Background on Petri nets}

In this section we recall the formalism used in the paper. For more details on Petri nets we address to [9].

A Place/Transition net $(\mathrm{P} / \mathrm{T}$ net $)$ is a structure $N=(P, T$, Pre, Post $)$, where $P$ is a set of $m$ places; $T$ is a set of $n$ transitions; Pre $: P \times T \rightarrow \mathbb{N}$ and Post $: P \times T \rightarrow \mathbb{N}$ are the pre- and post-incidence functions that specify the arcs; $C=$ Post - Pre is the incidence matrix. The preset and postset of a node $X \in P \cup T$ are denoted ${ }^{\bullet} X$ and $X^{\bullet}$ while ${ }^{\bullet} X^{\bullet}={ }^{\bullet} X \cup X^{\bullet}$.

A marking is a vector $M: P \rightarrow \mathbb{N}$ that assigns to each place of a $P / T$ net a non-negative integer number of tokens, represented by black dots. We denote $M(p)$ the marking of place $p$. A $P / T$ system or net system $\left\langle N, M_{0}\right\rangle$ is a net $N$ with an initial marking $M_{0}$.

A transition $t$ is enabled at $M$ iff $M \geq \operatorname{Pre}(\cdot, t)$ and may fire yielding the marking $M^{\prime}=$ $M+C(\cdot, t)$. We write $M[\sigma\rangle$ to denote that the sequence of transitions $\sigma$ is enabled at $M$, and we write $M[\sigma\rangle M^{\prime}$ to denote that the firing of $\sigma$ yields $M^{\prime}$. Note that in this paper we always assume that two or more transitions cannot simultaneously fire (non-concurrency hypothesis).

A marking $M$ is reachable in $\left\langle N, M_{0}\right\rangle$ iff there exists a firing sequence $\sigma$ such that $M_{0}[\sigma\rangle M$. 
The set of all markings reachable from $M_{0}$ defines the reachability set of $\left\langle N, M_{0}\right\rangle$ and is denoted $R\left(N, M_{0}\right)$.

A labeling function $L: T \rightarrow E$ assigns to each transition $t \in T$ a symbol from a given alphabet $E$. Note that the same label $e \in E$ may be associated to more than one transition while no transition may be labeled with the empty string $\lambda^{1}$. Using the notation of [4] and [11] we say that this labeling function is $\lambda$-free.

Definition 1 A Petri net system $\left\langle N, M_{0}\right\rangle$ with $\lambda$-free labeling function $L: T \rightarrow E$ is deterministic if for all markings $M \in R\left(N, M_{0}\right)$ and for any two transitions $t, t^{\prime} \in T$ :

$$
t \neq t^{\prime}, L(t)=L\left(t^{\prime}\right), M[t\rangle \Longrightarrow \neg M\left[t^{\prime}\right\rangle
$$

i.e., if two transitions are labeled with the same symbol they cannot simultaneously be enabled at $M$.

From the above definition it is clear that determinism is a behavioral property because it not only depends on the structure of the net, but on the initial marking as well. However, since in this paper we make no assumption on the initial marking, we also need to introduce a structural definition of determinism.

Definition 2 A Petri net $N$ with $\lambda$-free labeling function $L: T \rightarrow E$ is structurally deterministic if for any two transitions $t, t^{\prime} \in T$ :

$$
t \neq t^{\prime} \Longrightarrow L(t) \neq L\left(t^{\prime}\right)
$$

i.e., two different transitions cannot be labeled with the same symbol.

Note that if a Petri net $N$ is structurally deterministic, then the net system $\left\langle N, M_{0}\right\rangle$ is deterministic for all initial marking $M_{0}$.

In this paper we consider Petri nets that are not structurally deterministic. We say that a transition $t$ is nondeterministic if its label is also associated to other transitions, otherwise a transition $t$ is said to be deterministic. We also denote $T^{d}$ the set of deterministic transitions and $T^{n}$ the set of nondeterministic transitions. Clearly, $T=T^{d} \cup T^{n}$. For simplicity of notation, we assume that the transition enumeration is such that $T^{n}=\left\{t_{j} \mid j=1, \cdots, n^{n}\right\}$ and $T^{d}=$ $\left\{t_{j} \mid j=n^{n}+1, \cdots, n\right\}$, where $n^{n}=\left|T^{n}\right|$. Analogously, we say that an event $e$ is deterministic if there exists only one transition $t$ such that $L(t)=e$, otherwise we say that the event $e$ is nondeterministic. Therefore, with no ambiguity on the notation, we may write $E=E^{d} \cup E^{n}$.

\footnotetext{
${ }^{1}$ In the Petri net literature the empty string is denoted $\lambda$, while in the formal language literature it is denoted $\varepsilon$. In this paper we denote the empty string $\varepsilon$ but, for consistency with the Petri net literature, we still use the term $\lambda$-free for the labeling function.
} 
We denote as $T_{e}$ the set of transitions labeled $e$, i.e, $T_{e}=\{t \in T \mid L(t)=e\}$. Moreover, we denote as $\vec{s}_{e} \in\{0,1\}^{n}$ the characteristic vector of $T_{e}$, i.e., $\vec{s}_{e}(i)=1$ if $L\left(t_{i}\right)=e$, and $\vec{s}_{e}(i)=0$ otherwise.

We denote as $w$ the word of events associated to the sequence $\sigma$, i.e., $w=L(\sigma)$. Moreover, we denote as $\sigma_{0}$ the sequence of null length and $w_{0}$ the empty word. Finally, we use the notation $w_{i} \preccurlyeq w$ to denote the generic prefix of $w$ of length $i \leq k$, where $k$ is the length of $w$. In particular, for $i=0$, we have by definition the empty word, $w_{0}=\varepsilon$.

\section{Problem statement}

In this paper we deal with the problem of estimating the marking of a net system $\left\langle N, M_{0}\right\rangle$ whose marking cannot be directly observed. The following properties of the system will be assumed.

(A1) The structure of the net $N$ is known.

(A2) The initial marking $M_{0}$ is known.

(A3) Labels associated to transition firings can be observed.

After the word $w$ has been observed, we define the set $\mathcal{C}(w)$ of $w$-consistent markings as the set of all markings in which the system may be given the observed behavior.

Definition 3 Given an observed word $w$, the set of $w$-consistent markings is $\mathcal{C}(w)=\{M \in$ $\mathbb{N}^{m} \mid \exists$ a sequence of transitions $\sigma: M_{0}[\sigma\rangle M$ and $\left.L(\sigma)=w\right\}$.

Our goal is that of providing a systematic and efficient procedure to estimate the set of markings that are consistent with an observed word.

Clearly, $\mathcal{C}\left(w_{0}\right)=M_{0}$ and $\mathcal{C}(w)$ is a singleton if for all $e$ in $w, T_{e}$ is a singleton. On the contrary, the degree of nondeterminism may increase as the cardinality of $T_{e}$ increases.

Finally, let us observe through a simple example, that the cardinality of the set of consistent markings may either increase or decrease as the length of the observed word increases.

Example 4 Let us consider the Petri net system in Figure 1 where $T^{d}=\left\{t_{5}, t_{6}, t_{7}\right\}$ and $T^{n}=$ $\left\{t_{1}, t_{2}, t_{3}, t_{4}\right\}$. More precisely, $T_{a}=\left\{t_{1}, t_{2}\right\}, T_{b}=\left\{t_{3}, t_{4}\right\}, T_{c}=\left\{t_{5}\right\}, T_{d}=\left\{t_{6}\right\}$, and $T_{e}=\left\{t_{7}\right\}$.

Clearly when no event has been observed,

$$
\left.\mathcal{C}(\varepsilon)=\left\{\begin{array}{llllllll}
1 & 0 & 1 & 0 & 1 & 0 & 1 & 1
\end{array}\right]^{T}\right\} .
$$




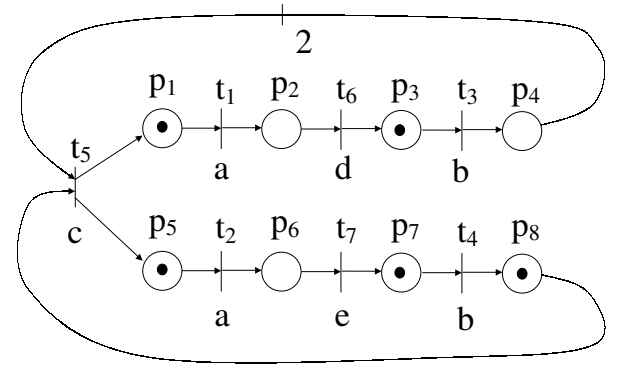

Figure 1: Petri net system that can only be partially observed

Let us first assume that the event $b$ is observed. Given the initial marking $M_{0}$, either $t_{3}$ or $t_{4}$ may have been fired, thus

$$
\mathcal{C}(b)=\left\{\left[\begin{array}{llllllll}
1 & 0 & 0 & 1 & 1 & 0 & 1 & 1
\end{array}\right]^{T},\left[\begin{array}{llllllll}
1 & 0 & 1 & 0 & 1 & 0 & 0 & 2
\end{array}\right]^{T}\right\} .
$$

Now, let $a$ be the next observed event. Label $a$ is associated to transitions $t_{1}$ and $t_{2}$ and both transitions are enabled at both markings in $\mathcal{C}(b)$. Therefore,

$$
\begin{aligned}
\mathcal{C}(b a)= & \left\{\begin{array}{llllllll}
0 & 1 & 0 & 1 & 1 & 0 & 1 & 1
\end{array}\right]^{T},\left[\begin{array}{llllllll}
1 & 0 & 0 & 1 & 0 & 1 & 1 & 1
\end{array}\right]^{T}, \\
& {\left.\left[\begin{array}{lllllllll}
0 & 1 & 1 & 0 & 1 & 0 & 0 & 2
\end{array}\right]^{T},\left[\begin{array}{llllllll}
1 & 0 & 1 & 0 & 0 & 1 & 0 & 2
\end{array}\right]^{T}\right\} . }
\end{aligned}
$$

Now, if the deterministic event $d$ is observed, we may be sure that neither $\left[\begin{array}{lllllll}1 & 0 & 0 & 1 & 0 & 1 & 1\end{array}\right]^{T}$ nor $\left[\begin{array}{llllllll}1 & 0 & 1 & 0 & 0 & 1 & 0 & 2\end{array}\right]^{T}$ in $\mathcal{C}(b a)$ may have been reached because none of these markings enables $t_{6}$. Thus, the set of markings consistent with $w=b a d$ is

$$
\mathcal{C}(\text { bad })=\left\{\left[\begin{array}{llllllll}
0 & 0 & 1 & 1 & 1 & 0 & 1 & 1
\end{array}\right]^{T},\left[\begin{array}{llllllll}
0 & 0 & 2 & 0 & 1 & 0 & 0 & 2
\end{array}\right]^{T}\right\}
$$

If $b$ is observed again, both transitions $t_{3}$ and $t_{4}$ may have fired from the first marking in $\mathcal{C}($ bad $)$, while only transition $t_{3}$ may have fired from the second marking. Thus

$$
\mathcal{C}(b a d b)=\left\{\left[\begin{array}{llllllll}
0 & 0 & 0 & 2 & 1 & 0 & 1 & 1
\end{array}\right]^{T},\left[\begin{array}{llllllll}
0 & 0 & 1 & 1 & 1 & 0 & 0 & 2
\end{array}\right]^{T}\right\} .
$$

Finally, if we observe the deterministic event $c$ we can conclude that only the first marking in $\mathcal{C}(b a d b)$ is compatible with the last observation, thus the actual marking of the net is completely reconstructed and

$$
\left.\mathcal{C}(\text { badbc })=\left\{\begin{array}{llllllll}
1 & 0 & 0 & 0 & 2 & 0 & 1 & 0
\end{array}\right]^{T}\right\} .
$$




\section{Computation of the set of consistent markings}

We first present a recursive algorithm strictly based on the definition of the set of consistent markings $\mathcal{C}(w)$, then we provide an algebraic characterization of $\mathcal{C}(w)$.

\section{Algorithm 5}

1. Let $\mathcal{C}\left(w_{0}\right)=M_{0}$.

2. Let $i=0$.

3. Wait until a new event $e$ is observed.

4. Let $i=i+1$.

5. Let $w_{i}=w_{i-1} e$.

6. Let $\mathcal{C}\left(w_{i}\right)=\emptyset$.

7. For all $M \in \mathcal{C}\left(w_{i-1}\right)$ do

For all $t$ such that $M[t\rangle$ and $L(t)=e$

compute $M^{\prime}=M+C(\cdot, t)$ and let $\mathcal{C}\left(w_{i}\right)=\mathcal{C}\left(w_{i}\right) \cup M^{\prime}$.

8. Goto 3.

Clearly, the main disadvantage of the above iterative algorithm is that to compute the set of markings that are consistent with an observed word $w$ of cardinality $k$, we preliminary need to compute the set of markings that are consistent with all prefixes $w_{i} \preccurlyeq w, i=1, \cdots, k-1$. Furthermore each set $\mathcal{C}\left(w_{i}\right)$ must be explicitly enumerated.

A better solution that does not require to enumerate the sets $\mathcal{C}\left(w_{i}\right)$ consists in using a linear algebraic characterization of the set of consistent markings.

Proposition 6 Let $\left\langle N, M_{0}\right\rangle$ be a net system and $w=e_{1}, \cdots, e_{k}$ be an observed word. The set of w-consistent markings is given by:

$$
\begin{aligned}
\mathcal{C}(w)=\left\{M^{(k)} \in \mathbb{N}^{m} \mid\right. & (\text { for all } i=1, \cdots k) \\
& \overrightarrow{1}^{T} \cdot \vec{\sigma}^{(i)}=1 \\
& \vec{s}_{e_{i}} \cdot \vec{\sigma}^{(i)}=1 \\
& M^{(i-1)} \geq \operatorname{Pre} \cdot \vec{\sigma}^{(i)} \\
& M^{(i)}=M^{(i-1)}+C \cdot \vec{\sigma}^{(i)} \\
& \left.\vec{\sigma}^{(i)} \in\{0,1\}^{n}\right\}
\end{aligned}
$$

where $M^{(0)}=M_{0}$ and $\overrightarrow{1}$ is the $n$-dimensional column vector of 1 's.

Proof: It follows from the definition of the set of consistent markings. In fact, for any observed event $e_{i}$, we introduce an unknown vector $\vec{\sigma}^{(i)}$ of zeros and ones (constraint (e)) representing the firing vector associated to the observed event. Then, the first constraint (a) imposes that 
when the event $e_{i}$ is observed, only one transition has fired and the second constraint (b) states that the label of that transition should be equal to the observed event. Moreover, if a transition has fired, then it should be enabled by at least one marking in the set $\mathcal{C}\left(w_{i-1}\right)$ (inequality (c)) and its firing brings to a new marking that is given by constraint (d).

Example 7 Let us consider again the net system depicted in Figure 1. Let us assume that the observed event is $b$. By virtue of Proposition 6 we may write:

$$
\begin{array}{rlr}
\mathcal{C}(b)=\left\{M^{(1)} \in \mathbb{N}^{8} \mid\right. & \overrightarrow{1}^{T} \cdot \vec{\sigma}^{(1)}=1 \\
& \sigma(3)^{(1)}+\sigma(4)^{(1)}=1 \\
& M^{(0)} \geq \operatorname{Pre} \cdot \vec{\sigma}^{(1)} & \left(b_{1}\right) \\
& M^{(1)}=M^{(0)}+C \cdot \vec{\sigma}^{(1)} \\
& \left.\vec{\sigma}^{(1)}=\{0,1\}^{7}\right\}
\end{array}
$$

where $M^{(0)}$ is the initial marking.

Now, let $a$ be the next observed event. Once again, using Proposition 6 we may conclude that the set of markings consistent with the observed word $w=b a$ is:

$$
\begin{array}{rlr}
\mathcal{C}(b a)=\left\{M^{(2)} \in \mathbb{N}^{8} \mid\right. & \overrightarrow{1}^{T} \cdot \vec{\sigma}^{(1)}=1 & \left(a_{1}\right) \\
& \sigma(3)^{(1)}+\sigma(4)^{(1)}=1 & \left(b_{1}\right) \\
& M^{(0)} \geq \operatorname{Pre} \cdot \vec{\sigma}^{(1)} & \left(c_{1}\right) \\
& M^{(1)}=M^{(0)}+C \cdot \vec{\sigma}^{(1)} & \left(d_{1}\right) \\
& \vec{\sigma}^{(1)}=\{0,1\}^{7} & \left(e_{1}\right) \\
& \overrightarrow{1}^{T} \cdot \vec{\sigma}^{(1)}=1 & \left(a_{2}\right) \\
& \sigma(1)^{(2)}+\sigma(2)^{(2)}=1 & \left(b_{2}\right) \\
& M^{(1)} \geq \operatorname{Pre} \cdot \vec{\sigma}^{(2)} & \left(c_{2}\right) \\
& M^{(2)}=M^{(1)}+C \cdot \vec{\sigma}^{(2)} \\
& \left.\vec{\sigma}^{(2)}=\{0,1\}^{7}\right\} & \left(d_{2}\right) \\
&
\end{array}
$$

This example clearly shows that, even if Proposition 6 enables us to directly describe the set of consistent markings without iterating on the sets of markings that are consistent with the prefixes of the observed word, it still presents a significant drawback. In fact, both the number of unknowns and the number of constraints increase as the length of the observed word increases.

The main goal of this paper is that of investigating whether it is possible to define the set of $w$-consistent markings using a fixed (even if large) number of constraints.

A general solution to this problem has not been determined yet. But the wide variety of scenarios we dealt with, enables us to conclude that this possibility is mainly related to the 
degree of contact of nondeterministic transitions. Moreover, it also depends on the number of transitions with the same label.

In the next section we present the first step of our research on this topic, consisting in the derivation of some restrictive assumptions under which it is possible to formally prove that the set of consistent markings may be expressed with a fixed number of constraints.

\section{The contact-free case}

In this section we assume that the following two conditions are verified:

(A4) for each label $e \in E$ there are at most two transitions such that $L(t)=e$, or equivalently, $\left|T_{e}\right| \leq 2$

(A5) nondeterministic transitions are contact free, i.e., for any two nondeterministic transitions $t_{i}$ and $t_{j}$, it holds that $\bullet_{i}^{\bullet} \cap t^{\bullet}=\emptyset$.

Note that, given assumption (A4), we always assume that the transition enumeration is such that $L\left(t_{r}\right)=L\left(t_{r+1}\right)$ for $r=1,3,5, \cdots n^{n}-1$.

In the following we formally prove that under the above assumptions, a fixed number of constraints, not depending on the length of the observed word $w$, may be used to describe the set of $w$ consistent markings. In particular, we formally prove that:

$$
\begin{array}{rlrl}
\mathcal{C}(w)=\left\{M \in \mathbb{N}^{m} \mid\right. & M=M_{0}+C \vec{\sigma}, & \\
& \sigma(r) \leq u_{r} & r=1,2, \cdots n^{n} \\
& \sigma(r)+\sigma(r+1)=n_{r} & r=1,3,5, \cdots n^{n}-1 & (\text { (b) } \\
& \sigma(q)=n_{q} & q=n^{n}+1, \cdots, n & (c) \\
& \left.\vec{\sigma} \in \mathbb{N}^{n}\right\} &
\end{array}
$$

is the set of $w$ consistent markings where the upper bounds $u_{r}$ 's are appropriately computed and $n_{r}\left(n_{q}\right)$ denotes the number of times a nondeterministic (deterministic) event $L\left(t_{r}\right)\left(L\left(t_{q}\right)\right)$ has been observed.

Note that any vector $\vec{\sigma}$ satisfying constraints (a) to (d) of eq. (3) represents an admissible firing vector associated to a sequence of transitions $\sigma$ that may have fired and whose labeling is equal to the observed word $w$, i.e., $L(\sigma)=w$.

For any couple of nondeterministic transitions $t_{r}$ and $t_{r+1}$ we have 3 constraints: for each transition we need an upper bound on the number of times it may have fired, plus an additional 
constraint keeping into account the total number of times the corresponding nondeterministic event $L\left(t_{r}\right)=L\left(t_{r+1}\right)$ has been observed $\left(n_{r}\right)$. On the contrary, for each deterministic transition $t_{q}$ we only need one constraint, because we exactly know how many times it has fired. In fact, in such a case $n_{q}$ is equal to the number of times the deterministic event $L\left(t_{q}\right)$ has been observed.

Looking at hypothesis (A4) and (A5) we may conclude that for each couple of nondeterministic transitions, the nets we are dealing with contain "nondeterministic" subnets whose structure is like that one shown in fig. 2, where weights associated to arcs are not required to be ordinary.

Now, let us present a simple algorithm that enables us to compute the upper bounds $u_{r}$ 's used in equation (3).

\section{Algorithm 8 (Upper bounds computation)}

1. Let $u_{r}=0$ for all $r=1, \cdots, n^{n}$.

2. Let $n_{q}=0$ for all $q=n^{n}+1, \cdots, n$.

3. Wait until an event $e$ is observed.

4. If $e \in E^{d}$, then

let $t_{q}$ be such that $t_{q} \in T^{d}$ and $L\left(t_{q}\right)=e$

$n_{q}=n_{q}+1$

if $t_{q} \in\left({ }^{\bullet} T^{n}\right)^{\bullet}$, then

for every $r \in\left\{1, \ldots, n^{n}\right\}$ such that $t_{r} \in\left(\bullet^{\bullet} t_{q}\right)^{\bullet}$, do

for all $p \in t_{r}$,

$$
\begin{aligned}
& M^{+}(p)=\sum_{t_{q} \in \bullet^{\bullet} \cap T^{d}} n_{q} \cdot \operatorname{Post}\left(p, t_{q}\right) \\
& M^{-}(p)=\sum_{t_{q} \in p \bullet \cap T^{d}} n_{q} \cdot \operatorname{Pre}\left(p, t_{q}\right)
\end{aligned}
$$

endfor

$$
\begin{aligned}
& z_{r}^{i n}=\left\lfloor\min _{p \in t_{r}}\left\{\frac{M_{0}(p)+M^{+}(p)-M^{-}(p)}{\operatorname{Pre}\left(p, t_{r}\right)}\right\}\right\rfloor \\
& u_{r}=\min \left(u_{r}, z_{r}^{i n}\right)
\end{aligned}
$$

endfor

endif

if $t_{q} \in\left(T^{n \bullet}\right)^{\bullet}$, then

for every $r \in\left\{1, \ldots, n^{n}\right\}$ such that $t_{r} \in \bullet^{\bullet}\left(t_{q}\right)$, do

for all $p \in t_{r}^{\bullet}$,

$$
\begin{aligned}
& M^{+}(p)=\sum_{t \in \bullet \text { } \cap \cap T^{d}} n_{q} \cdot \operatorname{Post}\left(p, t_{q}\right) \\
& M^{-}(p)=\sum_{t \in p^{\bullet} \cap T^{d}} n_{q} \cdot \operatorname{Pre}\left(p, t_{q}\right)
\end{aligned}
$$

endfor

$$
z_{r}^{\text {out }}=\left\lfloor\min _{p \in t_{r}}\left\{\frac{M^{-}(p)-M_{0}(p)-M^{+}(p)}{\operatorname{Post}\left(p, t_{r}\right)}\right\}\right\rfloor
$$




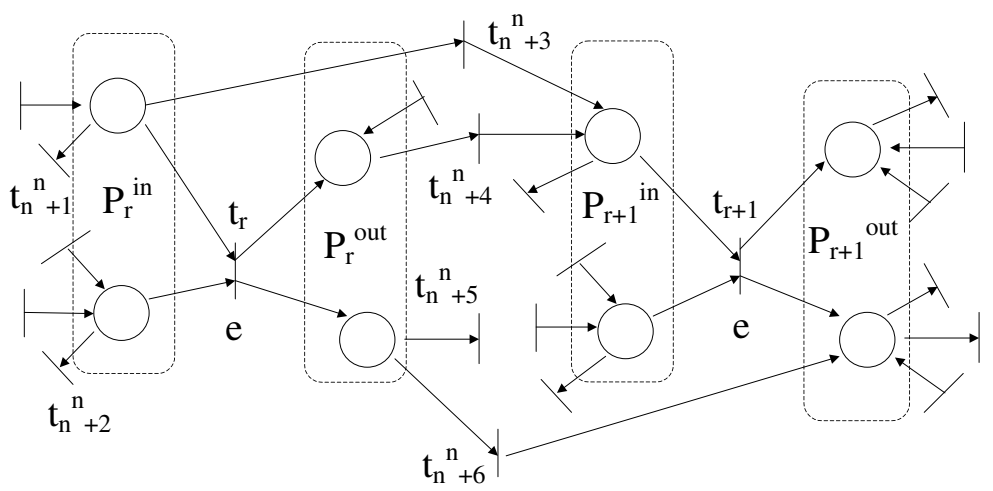

Figure 2: The generic couple of nondeterministic transitions $t_{r}$ and $t_{r+1}$.

$$
\begin{array}{r}
u_{\bar{r}}=\min \left(u_{\bar{r}}, n_{r}-z_{r}^{\text {out }}\right) \text { where } \bar{r}=r+1 \text { if } r \text { is odd, } \\
\text { else } \bar{r}=r-1
\end{array}
$$

endfor

endif

5. If $e \in E^{n}$ then

for every $r$ such that $L\left(t_{r}\right)=e$ do

for all $p \in \epsilon^{\bullet} t_{r}$

$$
\begin{aligned}
& M^{+}(p)=\sum_{t_{q} \in \bullet^{\prime} \cap T^{d}} n_{q} \cdot \operatorname{Post}\left(p, t_{q}\right) \\
& M^{-}(p)=\sum_{t_{q} \in p^{\bullet} \cap T^{d}} n_{q} \cdot \operatorname{Pre}\left(p, t_{q}\right)
\end{aligned}
$$

endfor

$$
\begin{gathered}
z_{r}^{i n}=\left\lfloor\min _{p \in \bullet_{r}}\left\{\frac{M_{0}(p)+M^{+}(p)-M^{-}(p)}{\operatorname{Pre}\left(p, t_{r}\right)}\right\}\right\rfloor \\
u_{r}=\min \left(u_{r}+1, z_{r}^{i n}\right)
\end{gathered}
$$

endfor

endif

6. Goto 3.

The main idea behind this algorithm is that of evaluating the upper bounds $u_{r}$ 's on the basis of the knowledge of two parameters associated to nondeterministic transitions. The first one is $z_{r}^{i n}$ that represents the enabling degree of transition $t_{r}$ assuming that it has never fired. This parameter is used to update the upper bound $u_{r}$ when one of the following two cases occur.

- If a deterministic transition $t_{q}$ fires and $t_{q} \in\left({ }^{\bullet} t_{r}\right)^{\bullet}\left(\right.$ see $t_{n^{n}+1}, t_{n^{n}+2}$ and $t_{n^{n}+3}$ in fig. 2), the value of $z_{r}^{i n}$ may decrease because we know for sure that some token(s) in $P_{r}^{i n}$ were still available to enable $t_{q}$. Thus, by definition of $z_{r}^{i n}$, we may conclude that $t_{r}$ may have fired at most $z_{r}^{i n}$ times. 
- A nondeterministic event $e$ is observed and $t_{r}$ is a transition whose label is $e$. In such a case, the value of $z_{r}^{i n}$ keeps the same and by definition of $z_{r}^{i n}$ we may conclude that $t_{r}$ may have fired at most $z_{r}^{i n}$ times.

The second parameter used to compute the upper bounds is $z_{r}^{\text {out }}$. It is a measure of the number of tokens that have been removed from the output places to $t_{r}$ by firing deterministic transitions exiting $P_{r}^{\text {out }}$ (see $t_{n^{n}+4}, t_{n^{n}+5}$ and $t_{n^{n}+6}$ in fig. 2). In particular, the value of $z_{r}^{\text {out }}$ is equal to the minimum number of times transition $t_{r}$ has to be fired to fulfill the token demands of the transitions exiting $P_{r}^{o u t}$. Consequently, it enables us to evaluate which is the maximum number of times transition $t_{r+1}$ may have fired, namely $u_{r+1}$. Analogously, the value of $z_{r+1}^{\text {out }}$ enables us to update the upper bound $u_{r}$.

Therefore the upper bounds associated to nondeterministic transitions may be updated when three different cases occur. This is clearly stated via the following simple example.

Example 9 Let us consider the ordinary Petri net system in Figure 3. There are only two nondeterministic transitions whose label is $a$.

The upper bounds $u_{1}$ and $u_{2}$ may be updated as a consequence of three different types of observed events.

1. Let us assume that the first observed event is $a$. Clearly, the upper bounds should be both updated to $u_{1}=u_{2}=1$ being $z_{1}^{i n}=z_{2}^{i n}=2$ and the initial bounds equal to zero. We are in the case of step 5 of Algorithm 8.

2. If the event $a$ is observed again, we are once again in the case of step 5 of Algorithm 8 . In particular, the upper bounds are both updated to $u_{1}=u_{2}=2$ being $z_{1}^{i n}=z_{2}^{i n}=2$ and the previous bounds equal to one.

Now, let us assume that $L\left(t_{3}\right)$ is observed, thus $n_{3}=1$ and $z_{1}^{i n}=1$. This means that for sure $t_{1}$ has fired at most one time, otherwise $t_{3}$ would have not been enabled. Thus the upper bound of $t_{1}$ is updated to $u_{1}=1$. We are in the first if case of step 4 of Algorithm 8 being $t_{3}$ an output transition to one input place of $t_{1}$.

3. Now, let us assume that $L\left(t_{8}\right)$ is observed, thus $w=a a L\left(t_{3}\right) L\left(t_{8}\right)$. This implies that $t_{1}$ should have fired at least once, and consequently $t_{2}$ should have fired at most once. In fact, in such a case $n_{8}=1, z_{1}^{\text {out }}=1$ and consequently $u_{2}=1$. We are in the second if case of step 4 of Algorithm 8.

Lemma 10 Let us consider a Petri net system $\left\langle N, M_{0}\right\rangle$ and let $L: T \rightarrow E$ be its labeling function. Assume that (A4) and (A5) are satisfied. Let $\mathcal{C}(w)$ be defined as in equation (3) where 


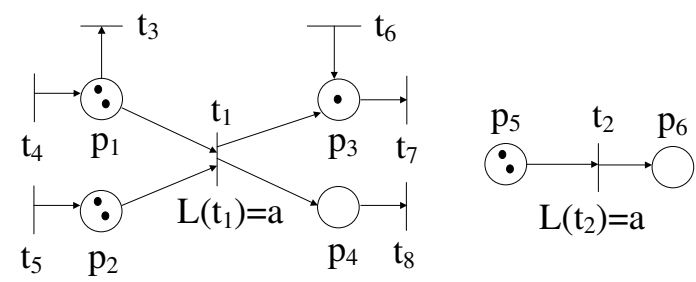

Figure 3: The Petri net system considered in example 9.

the upper bounds $u_{r}$ 's are computed using Algorithm 8. Assume that a label a is observed and there is a transition $t_{r}$ labeled $L\left(t_{r}\right)=a$ with bound $u_{r}$ such that it is disabled at any marking in $\mathcal{C}(w)$. Then the new bound $u_{r}^{\prime}$ computed by Algorithm 8 fulfills $u_{r}=u_{r}^{\prime}$.

Proof: First, notice that if transition $t_{r}$ is disabled at any marking in $\mathcal{C}(w)$ then all solutions of equation (3) verify $\sigma_{r}=z_{r}^{i n}$ where $z_{r}^{i n}$ is computed by Algorithm 8. In fact, $\sigma_{r}$ cannot be greater than $z_{r}^{i n}$ and being less would mean that there is a marking in $\mathcal{C}(w)$ in which $t_{r}$ is enabled. Furthermore $\sigma_{r}=u_{r}$ since if $\sigma_{r}<u_{r}$ then there would exist another solution for equation (3), let's say $\sigma_{r}^{\prime \prime}$, such that $\sigma_{r}^{\prime \prime}>\sigma_{r}$, meaning that $t_{r}$ was enabled at the consistent marking given by $\sigma_{r}$. Therefore we have $z_{r}^{i n}=u_{r}$ and since step 5 of Algorithm 8 computes $u_{r}^{\prime}$ as $u_{r}^{\prime}=\min \left(u_{r}+1, z_{r}^{i n}\right)$, we have $u_{r}^{\prime}=z_{r}^{i n}=u_{r}$.

Proposition 11 Let us consider a Petri net system $\left\langle N, M_{0}\right\rangle$ and let $L: T \rightarrow E$ be its labeling function. Let us assume that assumptions (A4) and (A5) are satisfied and let $w$ be an observed word of events. Then all markings in the set $\mathcal{C}(w)$ defined as in equation (3) are consistent with the observed word $w$, when the upper bounds ur's are computed using Algorithm 8.

Proof: We prove this by induction on the length of the observed word.

When no event is observed, i.e., when $w=w_{0}$ is the empty word, using equation (3) we have that $\mathcal{C}\left(w_{0}\right)=\left\{M_{0}\right\}$, thus the statement of the proposition holds.

Moreover, when a word $w_{k-1}$ of length $k-1$ is observed, we assume that all markings in $\mathcal{C}\left(w_{k-1}\right)$ are consistent with $w_{k-1}$, where $\mathcal{C}\left(w_{k-1}\right)$ is defined as in equation $(3)$ and the bounds are computed using Algorithm 8.

Now, let $e$ be a newly observed event, and let $w=w_{k}=w_{k-1} e$. We have to prove that all markings in $\mathcal{C}(w)$ are consistent with the observed word $w$.

For simplicity of presentation in the following we assume that there exists only one couple of nondeterministic transitions, thus $n^{n}=2$ and $n^{d}=n-2$. We call $a$ their label, i.e., $L\left(t_{1}\right)=L\left(t_{2}\right)=a$. Note that such an assumption does not affect the validity of the proof 
thanks to the contact freeness hypothesis (A5).

We partition the set of transitions as follows (see fig. 2):

$$
T=\bar{T} \cup T^{\text {in }} \cup T^{\text {out }} \cup T_{a}
$$

where $T_{a}=\left\{t_{1}, t_{2}\right\} ; P_{1}^{\text {in }}\left(P_{1}^{\text {out }}\right)$ and $P_{2}^{\text {in }}\left(P_{2}^{\text {out }}\right)$ are the set of input (output) places to transitions $t_{1}$ and $t_{2}$ respectively. $T^{i n}$ is the set of input and output transitions to $P_{1}^{i n}$ and $P_{2}^{i n}$, apart from $t_{1}$ and $t_{2} ; T^{\text {out }}$ is the set of input and output transitions to $P_{1}^{\text {out }}$ and $P_{2}^{\text {out }}$, apart from $t_{1}$ and $t_{2}$; finally, $\bar{T}$ is the set of deterministic transitions that are not contained in the previous sets.

Moreover, we define the following two sets ${ }^{2}$ :

$$
\mathcal{S}=\left\{\begin{array}{l}
\sigma_{1} \leq u_{1} \\
\sigma_{2} \leq u_{2} \\
\sigma_{1}+\sigma_{2}=n_{a} \\
\sigma_{1}, \sigma_{2} \in \mathbb{N}
\end{array} \quad \mathcal{S}^{\prime}=\left\{\begin{array}{l}
\sigma_{1} \leq u_{1}^{\prime} \\
\sigma_{2} \leq u_{2}^{\prime} \\
\sigma_{1}+\sigma_{2}=n_{a}^{\prime} \\
\sigma_{1}, \sigma_{2} \in \mathbb{N}
\end{array}\right.\right.
$$

where $\mathcal{S}\left(\mathcal{S}^{\prime}\right)$ consists of the subset of constraints of equation (3) only involving the nondeterministic transitions $t_{1}$ and $t_{2}$, when the observed word is $w_{k-1}(w)$. Clearly, these sets contain the only equations that are related to the nondeterministic part of the net, thus only an error on their definition may produce an error on the definition of the set of consistent markings. Therefore, the next step of the induction is proved if we demonstrate that each solution of $\mathcal{S}^{\prime}$ originates from a solution of $\mathcal{S}$ when the bounds are updated using Algorithm 8, i.e.,

- if the observed event is deterministic, i.e., $e \neq a$, then $\mathcal{S}^{\prime} \subseteq \mathcal{S}$;

- if the observed event is nondeterministic, i.e., $e=a$, then given a solution $\vec{\sigma}=\left(\sigma_{1}, \sigma_{2}\right) \in \mathcal{S}$, if $t_{1}$ (resp., $t_{2}$ ) is enabled from the marking corresponding to $\vec{\sigma}$, then $\vec{\sigma}^{\prime}=\left(\sigma_{1}+1, \sigma_{2}\right) \in \mathcal{S}^{\prime}$ (resp., $\left.\left(\sigma_{1}, \sigma_{2}+1\right) \in \mathcal{S}^{\prime}\right)$.

Now, when an event $e$ is observed, four different cases may occur.

1. A transition $t \in \bar{T}$ has fired. In such a case $\mathcal{S}^{\prime} \equiv \mathcal{S}$ and the statement of the proposition holds.

2. A transition $t \in T^{i n}$ has fired.

- a. If $t \in \bullet\left(P_{1}^{i n}\right) \cup \bullet\left(P_{2}^{i n}\right)$, no bound is updated thus $\mathcal{S}^{\prime} \equiv \mathcal{S}$.

- b. If $t \in\left(P_{1}^{i n}\right)^{\bullet} \cup\left(P_{2}^{i n}\right)^{\bullet}$ the upper bounds may either stay the same or may be even smaller thus $\mathcal{S}^{\prime} \subseteq \mathcal{S}$.

\footnotetext{
${ }^{2}$ Slightly abusing the notation, we denote with $\mathcal{S}$ and $\mathcal{S}^{\prime}$ both the set of constraints given by (5) and their respective solutions $\left(\sigma_{1}, \sigma_{2}\right)$.
} 
3. A transition $t \in T^{o u t}$ has fired.

- a. If $t \in \bullet\left(P_{1}^{\text {out }}\right) \cup \bullet\left(P_{2}^{\text {out }}\right)$, no bound is updated thus $\mathcal{S}^{\prime} \equiv \mathcal{S}$.

- b. If $t \in\left(P_{1}^{\text {out }}\right)^{\bullet} \cup\left(P_{2}^{\text {out }}\right)^{\bullet}$ the upper bounds may either stay the same or may be even smaller thus $\mathcal{S}^{\prime} \subseteq \mathcal{S}$.

4. A transition $t \in T_{a}$ has fired.

Let us denote $T_{a}^{e}$ the set of transitions whose label is $a$ and that are enabled by at least one marking in $\mathcal{C}\left(w_{k-1}\right)$. Two different cases may occur: (1) $T_{a}^{e}$ is a singleton, i.e., either $T_{a}^{e}=\left\{t_{1}\right\}$ or $T_{a}^{e}=\left\{t_{2}\right\}$. (2) $T_{a}^{e}=\left\{t_{1}, t_{2}\right\}$.

(1) With no loss of generality we may assume $T_{a}^{e}=\left\{t_{1}\right\}$. In such a case the generic solution $\left(\sigma_{1}^{\prime}, \sigma_{2}^{\prime}\right)$ of $\mathcal{S}^{\prime}$ may always be written as $\sigma_{1}^{\prime}=\tilde{\sigma}_{1}+1, \sigma_{2}^{\prime}=\tilde{\sigma}_{2}$. In fact, if this was not possible, then $\sigma_{1}^{\prime}=0$ and $\sigma_{2}^{\prime}=n_{a}^{\prime}=n_{a}+1>n_{a} \geq u_{2}=u_{2}^{\prime}$, where the last equality follows from lemma 10. Therefore, we would obtain $\sigma_{2}^{\prime}>u_{2}^{\prime}$, that leads to a contradiction.

Now, we want to prove that $\left(\tilde{\sigma}_{1}, \tilde{\sigma}_{2}\right)$ is a solution of $\mathcal{S}$. By simply substituting $\left(\sigma_{1}^{\prime}, \sigma_{2}^{\prime}\right)$ in (5) where $\mathcal{S}^{\prime}$ is defined, and taking into account that $n_{a}^{\prime}=n_{a}+1, u_{2}^{\prime}=u_{2}$ and $u_{1}^{\prime}=u_{1}+1$, we can trivially verify that $\left(\tilde{\sigma}_{1}, \tilde{\sigma}_{2}\right) \in \mathcal{S}$.

(2) Let us now consider the case in which $T_{a}^{e}=\left\{t_{1}, t_{2}\right\}$. We first observe that for at least one transition $t_{i} \in T_{a}^{e}, \sigma_{i}^{\prime}>\sigma_{i}^{\text {min }}$, where $\sigma_{i}^{\text {min }}, i=1,2$, is the minimum value of $\sigma_{i}$ for any $\left(\sigma_{1}, \sigma_{2}\right) \in \mathcal{S}$. In fact, if this was not true, then for all solutions $\left(\sigma_{1}, \sigma_{2}\right) \in \mathcal{S}$, and $\left(\sigma_{1}^{\prime}, \sigma_{2}^{\prime}\right) \in \mathcal{S}^{\prime}$ it holds that $n_{a}^{\prime}=\sigma_{1}^{\prime}+\sigma_{2}^{\prime}=\sigma_{1}^{\text {min }}+\sigma_{2}^{\text {min }} \leq \sigma_{1}+\sigma_{2}=n_{a}$ contradicting $n_{a}^{\prime}=n_{a}+1>n_{a}$.

Now, with no loss of generality we assume that $\sigma_{1}^{\prime}>\sigma_{1}^{\text {min }} \geq 0$. Then, we may write $\tilde{\sigma}_{1}=\sigma_{1}^{\prime}-1$ and $\tilde{\sigma}_{2}=\sigma_{2}^{\prime}$. We show that $\left(\tilde{\sigma}_{1}, \tilde{\sigma}_{2}\right) \in \mathcal{S}$.

The only constraint that is not trivially verified is $\tilde{\sigma}_{2} \leq u_{2}$. In fact, $\sigma_{2}^{\prime} \leq u_{2}^{\prime} \rightarrow$ $\tilde{\sigma}_{2} \leq u_{2}^{\prime}$. However, we show that if $\sigma_{2}^{\prime}=u_{2}^{\prime}=u_{2}+1$ then $\sigma_{1}^{\prime}=n_{a}^{\prime}-u_{2}^{\prime}=n_{a}+$ $1-u_{2}-1=n_{a}-u_{2}$. By assumption $\sigma_{1}^{\prime}>\sigma_{1}^{\text {min }}$, thus $\sigma_{1}^{\prime}>n_{a}-u_{2}$ that leads to a contradiction.

Proposition 12 Let us consider a net system $\left\langle N, M_{0}\right\rangle$ and let $L: T \rightarrow E$ be its labeling function. Let us assume that assumptions (A4) and (A5) are satisfied and let $w$ be an observed word of events. Then all markings that are consistent with the observed word $w$ are contained in $\mathcal{C}(w)$, when $\mathcal{C}(w)$ is defined as in equation (3) and the upper bounds $u_{r}$ 's are computed using Algorithm 8. 
Proof: We prove this by induction on the length of the observed word. Clearly, when no event is observed the only consistent marking is the initial one, thus the statement of the proposition holds. Moreover, we assume that it also holds when a word $w_{k-1}$ is observed, i.e., we assume that there exists no marking that is consistent with $w_{k-1}$ and that is not contained in $\mathcal{C}\left(w_{k-1}\right)$.

To complete the proof, we must demonstrate that when a new event $e$ is observed, i.e., when the current word is $w=w_{k}=w_{k-1} e$, all markings that are consistent with $w$ are contained in $\mathcal{C}(w)$. As in the case of the previous proposition, thanks to the contact freeness assumption (A5), we may assume that there exists only one couple of nondeterministic transitions, namely $t_{1}$ and $t_{2}$. Therefore, we may restrict our attention to the sets $\mathcal{S}$ and $\mathcal{S}^{\prime}$ defined in equation (5). Now, the next step of the induction is proved if we demonstrate that, from each solution $\left(\sigma_{1}, \sigma_{2}\right) \in \mathcal{S}$ corresponding to a marking in $\mathcal{C}\left(w_{k-1}\right)$ enabling a transition labeled $e$, we get a solution $\left(\sigma_{1}^{\prime}, \sigma_{2}^{\prime}\right) \in \mathcal{S}^{\prime}$ that is a consistent marking associated to the observation of $e$.

We refer again to the partition of $T$ introduced via equation (4) and we consider four different cases.

1. A transition $t \in \bar{T}$ fires. Being $\mathcal{S}^{\prime} \equiv \mathcal{S}$, the statement of the proposition is trivially verified.

2. A transition $t \in T^{i n}$ fires. In such a case, $\mathcal{S}^{\prime} \subseteq \mathcal{S}$ and we must prove that when updating the bounds we are not neglecting markings that are consistent with $w$. However, by looking at Algorithm 8 we may observe that $\mathcal{S}^{\prime} \subset \mathcal{S}$ if and only if $\exists r \in\{1,2\}$ such that $t \in\left({ }^{\bullet} t_{r}\right)^{\bullet}$ and $z_{r}^{i n}<u_{r}$ (first if case of step 4 of Algorithm 8). But this is correct because if we allow $u_{r}^{\prime}$ to be greater than $z_{r}^{i n}$, the non-negativity constraints would be violated.

3. A transition $t \in T^{\text {out }}$ fires. This case is similar to the previous one. In fact, $\mathcal{S}^{\prime} \subseteq \mathcal{S}$. In particular, $\mathcal{S}^{\prime} \subset \mathcal{S}$ if and only if $\exists r \in\{1,2\}$ such that $t \in\left(t_{r}^{\bullet}\right)^{\bullet}$ and $n_{r}-z_{r}^{\text {out }}<u_{\bar{r}}$, where $\bar{r}$ is defined as in step 4 of Algorithm 8. But this is correct, because $z_{r}^{\text {out }}$ denotes by definition the number of times transition $t_{r}$ has fired for sure. If we allow $u_{\bar{r}}$ to be greater than $n_{r}-z_{r}^{\text {out }}$ (or equivalently $u_{r}$ to be smaller than $z_{r}^{\text {out }}$ ), the non-negativity constraints are violated.

4. A transition $t \in T^{a}$ fires. We must prove that, given a solution $\vec{\sigma}=\left(\sigma_{1}, \sigma_{2}\right) \in \mathcal{S}$, if $t_{1}$ (resp., $t_{2}$ ) is enabled from the marking corresponding to $\vec{\sigma}$, then $\vec{\sigma}^{\prime}=\left(\sigma_{1}+1, \sigma_{2}\right) \in \mathcal{S}^{\prime}$ (resp., $\left.\left(\sigma_{1}, \sigma_{2}+1\right) \in \mathcal{S}^{\prime}\right)$.

With no loss of generality we may assume that $t_{1}$ is enabled from the marking corresponding to $\vec{\sigma}$. This implies that for that $\vec{\sigma}$ it holds that $\sigma_{1}<z_{r}^{i n}$ being by definition $z_{r}^{i n}$ the enabling degree of transition $t_{r}$ assuming that $t_{r}$ has never fired. Thus, $\sigma_{1}<z_{r}^{i n}, \quad \sigma_{1} \leq u_{r} \quad \Longrightarrow \sigma_{1}^{\prime}=\sigma_{1}+1 \leq \min \left(u_{r}+1, z_{r}^{i n}\right)=u_{1}^{\prime}$. 
Moreover, $\sigma_{1}^{\prime}-1+\sigma_{2}^{\prime}=n_{a} \rightarrow \sigma_{1}^{\prime}+\sigma_{2}^{\prime}=n_{a}^{\prime}$. Therefore, we may conclude that $\left(\sigma_{1}^{\prime}, \sigma_{2}^{\prime}\right) \in \mathcal{S}^{\prime}$.

Theorem 13 Let us consider a net system $\left\langle N, M_{0}\right\rangle$ and let $L: T \rightarrow E$ be its labeling function. Let us assume that assumptions (A4) and (A5) are satisfied and let $w$ be an observed word of events. Then the set $\mathcal{C}(w)$ defined by equation (3) contains all and only those markings that are consistent with the observed word $w$, when the upper bounds $u_{r}$ 's are computed using Algorithm 8.

Proof: It follows from Propositions 11 and 12.

Example 14 Let us consider again the Petri net system in Figure 1. Let us first observe that assumptions (A4) and (A5) are verified. Therefore, by virtue of Theorem 13, the set of consistent markings can be described in terms of equation (3) where the upper bounds are computed using Algorithm 8.

All bounds are initially set to zero, thus the set of markings consistent with the empty word is

$$
\begin{aligned}
\mathcal{C}(\varepsilon)=\left\{M \in \mathbb{N}^{8} \mid\right. & M=M_{0}+C \vec{\sigma}, \\
& \sigma\left(t_{1}\right), \sigma\left(t_{2}\right), \sigma\left(t_{3}\right), \sigma\left(t_{4}\right) \leq 0 \\
& \sigma\left(t_{1}\right)+\sigma\left(t_{2}\right)=0 \\
& \sigma\left(t_{3}\right)+\sigma\left(t_{4}\right)=1 \\
& \sigma\left(t_{5}\right)=\sigma\left(t_{6}\right)=\sigma\left(t_{7}\right)=0 \\
& \left.\vec{\sigma} \in \mathbb{N}^{7}\right\}
\end{aligned}
$$

and the only admissible firing vector is $\vec{\sigma}=\overrightarrow{0}$, i.e., the only consistent marking is the initial one.

Now, assume that the nondeterministic event $b$ is observed. In such a case both $z_{3}^{i n}$ and $z_{4}^{i n}$ are set to one (see step 5 of Algorithm 8), thus $u_{3}$ and $u_{4}$ are updated to one. On the contrary, all the other bounds keep equal to zero. Therefore,

$$
\begin{aligned}
\mathcal{C}(b)=\left\{M \in \mathbb{N}^{8} \mid\right. & M=M_{0}+C \vec{\sigma}, \\
& \sigma\left(t_{1}\right) \leq 0 \\
& \sigma\left(t_{2}\right) \leq 0 \\
& \sigma\left(t_{3}\right) \leq 1 \\
& \sigma\left(t_{4}\right) \leq 1 \\
& \sigma\left(t_{1}\right)+\sigma\left(t_{2}\right)=0 \\
& \sigma\left(t_{3}\right)+\sigma\left(t_{4}\right)=1 \\
& \sigma\left(t_{5}\right)=\sigma\left(t_{6}\right)=\sigma\left(t_{7}\right)=0 \\
& \left.\vec{\sigma} \in \mathbb{N}^{7}\right\}
\end{aligned}
$$


It is easy to verify that in this case there are two admissible firing vectors, namely, $\vec{\sigma}_{1}=$ $\left[\begin{array}{lllllll}0 & 0 & 1 & 0 & 0 & 0 & 0\end{array}\right]^{T}$ (corresponding to the firing of $t_{3}$ ) and $\vec{\sigma}_{2}=\left[\begin{array}{lllllll}0 & 0 & 0 & 1 & 0 & 0 & 0\end{array}\right]^{T}$ (corresponding to the firing of $\left.t_{4}\right)$. In terms of consistent markings, this means that

$$
\mathcal{C}(b)=\left\{\left[\begin{array}{llllllll}
1 & 0 & 0 & 1 & 1 & 0 & 1 & 1
\end{array}\right]^{T},\left[\begin{array}{llllllll}
1 & 0 & 1 & 0 & 1 & 0 & 0 & 2
\end{array}\right]^{T}\right\} .
$$

Similarly, if the nondeterministic event $a$ is observed, we get $u_{1}=u_{2}=1$ and the set of consistent markings can be written as

$$
\begin{aligned}
& \mathcal{C}(b a)=\left\{M \in \mathbb{N}^{8} \mid\right. M=M_{0}+C \vec{\sigma}, \\
& \sigma\left(t_{1}\right) \leq 1 \\
& \sigma\left(t_{2}\right) \leq 1 \\
& \sigma\left(t_{3}\right) \leq 1 \\
& \sigma\left(t_{4}\right) \leq 1 \\
& \sigma\left(t_{1}\right)+\sigma\left(t_{2}\right)=1 \\
& \sigma\left(t_{3}\right)+\sigma\left(t_{4}\right)=1 \\
& \sigma\left(t_{5}\right)=\sigma\left(t_{6}\right)=\sigma\left(t_{7}\right)=0 \\
&\left.\vec{\sigma} \in \mathbb{N}^{7}\right\}
\end{aligned}
$$

This implies that there are four admissible firing vectors, namely, $\vec{\sigma}_{1}=\left[\begin{array}{llllll}1 & 0 & 1 & 0 & 0 & 0\end{array}\right]^{T}, \vec{\sigma}_{2}=$ $\left[\begin{array}{lllllll}1 & 0 & 0 & 1 & 0 & 0 & 0\end{array}\right]^{T}, \vec{\sigma}_{3}=\left[\begin{array}{lllllll}0 & 1 & 1 & 0 & 0 & 0 & 0\end{array}\right]^{T}$, and $\vec{\sigma}_{4}=\left[\begin{array}{lllllll}0 & 1 & 0 & 1 & 0 & 0 & 0\end{array}\right]^{T}$. In terms of consistent markings this means that

$$
\begin{aligned}
\mathcal{C}(b a)= & \left\{\begin{array}{llllllll}
0 & 1 & 0 & 1 & 1 & 0 & 1 & 1
\end{array}\right]^{T},\left[\begin{array}{llllllll}
1 & 0 & 0 & 1 & 0 & 1 & 1 & 1
\end{array}\right]^{T} \\
& {\left.\left[\begin{array}{llllllll}
0 & 1 & 1 & 0 & 1 & 0 & 0 & 2
\end{array}\right]^{T},\left[\begin{array}{lllllllll}
1 & 0 & 1 & 0 & 0 & 1 & 0 & 2
\end{array}\right]^{T}\right\} . }
\end{aligned}
$$

Now, if the deterministic event $d$ is observed, we have that $z_{1}^{\text {out }}=1$ and consequently the upper bound associated to $t_{2}$ is set to $u_{2}=0$. The set of consistent markings is thus

$$
\begin{aligned}
& \mathcal{C}(\text { bad })=\left\{M \in \mathbb{N}^{8} \mid M=M_{0}+C \vec{\sigma},\right. \\
& \sigma\left(t_{1}\right) \leq 1 \quad(a 1) \\
& \sigma\left(t_{2}\right) \leq 0 \quad(a 2) \\
& \sigma\left(t_{3}\right) \leq 1 \\
& \sigma\left(t_{4}\right) \leq 1 \\
& \sigma\left(t_{1}\right)+\sigma\left(t_{2}\right)=1 \quad(b 1) \\
& \sigma\left(t_{3}\right)+\sigma\left(t_{4}\right)=1 \quad(b 2) \\
& \sigma\left(t_{6}\right)=1 \quad(c 1) \\
& \sigma\left(t_{5}\right)=\sigma\left(t_{7}\right)=0 \quad(c 2) \\
& \left.\vec{\sigma} \in \mathbb{N}^{7}\right\}
\end{aligned}
$$




\begin{tabular}{|l||c|c|c|c|c|c|c|c|c|c|c|c|}
\hline$\omega$ & $z_{1}^{\text {in }}$ & $z_{2}^{\text {in }}$ & $z_{3}^{\text {in }}$ & $z_{4}^{\text {in }}$ & $z_{1}^{\text {out }}$ & $z_{2}^{\text {out }}$ & $z_{3}^{\text {out }}$ & $z_{4}^{\text {out }}$ & $u_{1}$ & $u_{2}$ & $u_{3}$ & $u_{4}$ \\
\hline \hline$b$ & 1 & 1 & 1 & 1 & 0 & 0 & 0 & 0 & 0 & 0 & 1 & 1 \\
\hline$b a$ & 1 & 1 & 1 & 1 & 0 & 0 & 0 & 0 & 1 & 1 & 1 & 1 \\
\hline$b a d$ & 1 & 1 & 1 & 1 & 1 & 0 & 0 & 0 & 1 & 0 & 1 & 1 \\
\hline$b a d b$ & 1 & 1 & 2 & 1 & 1 & 0 & 0 & 0 & 1 & 0 & 2 & 1 \\
\hline$b a d b c$ & 1 & 1 & 2 & 1 & 1 & 0 & 2 & 0 & 1 & 0 & 2 & 0 \\
\hline
\end{tabular}

Table 1: The numerical results of example 14.

Finally, if we assume that the whole observed word is $w=b a d b c$, we obtain the results briefly summarized in table 1. This means that after the observation of $c$ the marking is perfectly known because there exists one admissible firing vector, namely $\vec{\sigma}=\left[\begin{array}{lllllll}1 & 0 & 2 & 0 & 1 & 1 & 0\end{array}\right]^{T}$.

Clearly these results are coincident with those presented in Section 3.

As a final remark, we want to stress the fact that the contact-freeness assumption is an essential requirement for the soundness of our approach. To make this point clear, we present the following very simple example.

Example 15 Let us consider the Petri net system in Figure 4 whose initial marking is $M_{0}=$ $\left[\begin{array}{ll}2 & 2\end{array}\right]^{T}$. It is immediate to observe that the two nondeterministic transitions are not contact-free.

Assume that the event $a$ is observed. Using Algorithm 8, we obtain that

$$
\begin{aligned}
& \mathcal{C}(a)=\left\{M \in \mathbb{N}^{2} \mid\right. M=M_{0}+C \vec{\sigma}, \\
& \sigma\left(t_{1}\right) \leq 1 \\
& \sigma\left(t_{2}\right) \leq 1 \\
& \sigma\left(t_{1}\right)+\sigma\left(t_{2}\right)=1 \\
& \sigma\left(t_{3}\right)=0 \\
&\left.\vec{\sigma} \in \mathbb{N}^{3}\right\}
\end{aligned}
$$

Now, assume that the event $a$ is observed again. In such a case the above set is updated to

$$
\begin{aligned}
& \mathcal{C}(a a)=\left\{M \in \mathbb{N}^{2} \mid\right. M=M_{0}+C \vec{\sigma}, \\
& \sigma\left(t_{1}\right) \leq 1 \\
& \sigma\left(t_{2}\right) \leq 2 \\
& \sigma\left(t_{1}\right)+\sigma\left(t_{2}\right)=2 \\
& \sigma\left(t_{3}\right)=0 \\
&\left.\vec{\sigma} \in \mathbb{N}^{3}\right\}
\end{aligned}
$$




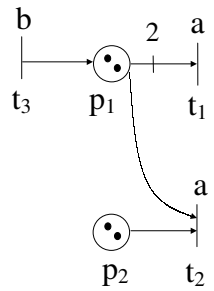

Figure 4: The Petri net system considered in example 15.

Finally, assume that the deterministic transition $t_{3}$ fires, thus

$$
\begin{aligned}
\mathcal{C}(a a b)=\left\{M \in \mathbb{N}^{2} \mid\right. & M=M_{0}+C \vec{\sigma}, \\
& \sigma\left(t_{1}\right) \leq 1 \\
& \sigma\left(t_{2}\right) \leq 2 \\
& \sigma\left(t_{1}\right)+\sigma\left(t_{2}\right)=2 \\
& \sigma\left(t_{3}\right)=1 \\
& \left.\vec{\sigma} \in \mathbb{N}^{3}\right\}
\end{aligned}
$$

The marking $M=\left[\begin{array}{ll}1 & 1\end{array}\right]^{T}$ obtained by firing $\vec{\sigma}=\left[\begin{array}{lll}1 & 1 & 1\end{array}\right]^{T}$ at the initial marking is assumed to be consistent with the actual observation, while it is a spurious solution. In fact, $\vec{\sigma}=\left[\begin{array}{lll}1 & 1 & 1\end{array}\right]^{T}$ is not an admissible firing vector at $M_{0}$.

In such a case the only marking that is consistent with the word $w=a a b$ is $M=\left[\begin{array}{ll}1 & 0\end{array}\right]^{T}$ because the only admissible firing vector is $\vec{\sigma}^{\prime}=\left[\begin{array}{lll}0 & 2 & 1\end{array}\right]^{T}$.

This clearly shows that in this case, where the contact-freeness assumption is not satisfied, the proposed characterization of the set of consistent markings, when the upper bounds are computed using Algorithm 8, is no more valid.

\section{Conclusions}

We have presented a marking estimation procedure that can be applied to labeled Petri nets. Under some assumptions, we proved that the markings consistent with an observed sequence can be described by a constraint set of linear inequalites: this set has a fixed structure that does not change as the length of the observed sequence increases.

We plan to extend our results in several ways.

Firstly, we believe it may be possible to modify the structure of the constraint set to also take into account the case that the initial marking is not known. 
Secondly, it may also be possible to relax the assumption that at most two transitions may share the same label.

Finally, we plan to extend this approach to arbitrary labeling functions, i.e., functions $L$ : $T \rightarrow E \cup\{\varepsilon\}$ that may assign to one or more transitions the empty string $\varepsilon$. Transitions labeled by $\varepsilon$ are called silent (or unobservable) because their firing cannot be detected.

\section{References}

[1] A. Benasser, (2000). "Reachability in Petri nets: an approach based on constraint programming", Ph.D. Thesis, Université de Lille, France (in French).

[2] P.E. Caines, R. Greiner, S. Wang, (1988). "Dynamical logic observers for finite automata", 27th Conf. on Decision and Control, Austin, Texas, pp. 226-233.

[3] P.E. Caines, S. Wang. (1989). "Classical and logic based regulator design and its complexity for partially observed automata", 28th Int. Conf. on Decision and Control, Tampa, Florida, pp. $132-137$.

[4] S. Gaubert, A. Giua. (1999). "Petri net languages and infinite subsets of $\mathbb{N}^{m}$ ", Journal of Computer and System Sciences, Vol. 59, No. 3, pp. 373-391.

[5] A. Giua. (1997). "Petri net state estimators based on event observation", 36th Int. Conf. on Decision and Control, San Diego, California, pp. 4086-4091.

[6] A. Giua, C., Seatzu. (2002). "Observability of place/transition nets", IEEE Trans. on Automatic Control, Vol. 47, No. 9, pp. 1424-1437.

[7] R. Kumar, V. Garg, S.I. Markus. (1993). "Predicates and predicate transformers for supervisory control of discrete event dynamical systems", IEEE Trans. on Automatic Control, Vol. 38, No. 2, pp. 232-247.

[8] M.E. Meda, A. Ramírez, A. Malo. (1998). "Identification in discrete event systems", IEEE Int. Conf. on Systems, Man and Cybernetics, San Diego, California, pp. 740-745.

[9] T. Murata. (1989). "Petri nets: properties, analysis and applications", Proc. IEEE, Vol. 77, No. 4 , pp. 541-580.

[10] C.M. Özveren, A.S. Willsky. (1990). "Observability of discrete event dynamic systems", IEEE Trans. on Automatic Control, Vol. 35, No. 7, pp. 797-806.

[11] J.L. Peterson. (1981). "Petri net theory and the modeling of systems", Prentice-Hall, 1981. 
[12] P.J. Ramadge. (1986). "Observability of discrete-event systems", 25th Conf. on Decision and Control, Athens, Greece, pp. 1108-1112. 